\title{
ON THE REALIZABILITY OF MODULES OVER THE STEENROD ALGEBRA
}

\author{
BY H. R. MARGOLIS
}

Communicated by E. H. Brown, Jr., November 29, 1971

1. Introduction. Let $A$ denote the mod 2 Steenrod algebra. There is good reason to believe that knowledge both of the structure of the category of $A$-modules and of the subcategory of those $A$-modules which are the cohomology of spectra (i.e. are realizable), will give us great insight into the structure of the stable homotopy category of spaces. So far the structure and realizability problems have received little attention of a general nature, work having mostly centered around the specific insights needed to attack specific problems. For example, with respect to the realizability problem, research to date gives us little more than the realizability of specific $A$-modules (e.g. [2], [3] and [4]).

This paper describes a step in the attack of these problems from a more general point of view.

I begin by recalling work by J. F. Adams and myself [1] concerning the structure problem. Using this work I describe a family of natural constructions analogous to the killing of homotopy groups. These constructions can be performed both on $A$-modules and on spectra, and under mod 2 cohomology they correspond. Therefore if an $A$-module is realizable then so are the modules obtained from it by the constructions.

A more detailed exposition of this work and its ramifications is in preparation.

2. Modules over the Steenrod algebra. The structure of the mod 2 Steenrod algebra, $A$, is well known and we are in particular interested in a family of elements best describable in terms of the Milnor basis [5]: Let $P_{t}^{s}$ be the dual of $\xi_{t}^{2 s}$. Then an easy computation using the product formula gives us that $\left(P_{t}^{s}\right)^{2}=0$ if $s<t$. This allows the definition:

Definition 2.1. Let $M$ be a left $A$-module; if $s<t$ then define $H\left(M, P_{t}^{s}\right)=\operatorname{ker} P_{t}^{s} \mid M / P_{t}^{s} M$, the $P_{t}^{s}$-homology group of $M$. This is clearly a functorial invariant of $M$. It is also graded by the grading on $M$. In what follows we will only consider $P_{t}^{s}$ with $s<t$.

These are strong invariants as evidenced by the following:

TheOREM 2.2 [1]. If $f: M \rightarrow N$ is a map of bounded below (b.b.) A-modules and $H\left(f, P_{t}^{s}\right)$ is an isomorphism for all $s<t$ then $M$ and $N$ are stably iso-

AMS 1970 subject classifications. Primary 55G10, 55D99.

Key words and phrases. Steenrod algebra, spectra, realizability.

Copyright (C) American Mathematical Society 1972 
morphic, i.e. there are free $A$-modules $F$ and $G$ such that $M \oplus F$ and $N \oplus G$ are isomorphic.

They are also very well behaved and in particular we have the following results needed in $\$ 3$.

THEOREM 2.3. Let $B$ be a sub-Hopf algebra of $A$ and let $M=A \otimes_{B} N$ for a $B$-module $N$. If $P_{t}^{s} \notin B$ then $H\left(M, P_{t}^{s}\right)=0$ and if $P_{t}^{s} \in B$ there is a spectral sequence with $E^{1}=\left(A \otimes_{B} Z_{2}\right) \otimes H\left(N, P_{t}^{s}\right)$ and $E^{\infty}=H\left(M, P_{t}^{s}\right)$.

THEOREM 2.4. Given $f \otimes g: M_{1} \otimes M_{2} \rightarrow N_{1} \otimes N_{2} ;$ if $H\left(f, P_{t}^{s}\right)$ and $H\left(g, P_{t}^{s}\right)$ are isomorphisms then $H\left(f \otimes g, P_{t}^{s}\right)$ is an isomorphism.

3. Killing homology groups. Let $X^{*}$ denote $H^{*}\left(X ; Z_{2}\right)$-this conforms pleasantly with the usual notation $f^{*}$ for maps. The homology groups of $\$ 2$ give us invariants of spectra if we consider $H\left(X^{*}, P_{t}^{s}\right)$. These invariants have the same strength in the topological setting as they did in the algebraic:

THEOREM 3.1. Let $f: X \rightarrow Y$ be a map of b.b. spectra such that $H\left(f^{*}, P_{t}^{s}\right)$ is an isomorphism for all $P_{t}^{s}$, then $X$ and $Y$ are stably equivalent, i.e. homotopy equivalent up to $K\left(Z_{2}\right)$ factors.

The constructions alluded to in $\$ 1$ play the same role for the homology groups as the constructions of "killing homotopy groups" play for homotopy groups. The implied ordering is not the grading of an individual homology group but the ordering by degree of the $P_{t}^{s}$ 's. Note that $P_{t_{1}}^{s_{1}} \neq P_{t_{2}}^{s_{2}}$ implies $\operatorname{deg} P_{t_{1}}^{s_{1}} \neq \operatorname{deg} P_{t_{2}}^{s_{2}}$ so this ordering is linear.

DEFINITION 3.2. Let $X$ be a spectrum, then a spectrum $Y$ is of type $X\left\langle 0, P_{t}^{s}\right\rangle$ (resp. type $X\left\langle P_{t}^{s}, \infty\right\rangle$ ) if there is a map $f: X \rightarrow Y$ (resp. $f: Y \rightarrow X$ ) such that $H\left(f^{*}, P_{t_{1}}^{s_{1}}\right)$ is an isomorphism if $\operatorname{deg} P_{t_{1}}^{s_{1}} \leqq \operatorname{deg} P_{t}^{s}\left(\right.$ resp. $\operatorname{deg} P_{t_{1}}^{s_{1}}$ $\left.\geqq \operatorname{deg} P_{t}^{s}\right)$ and $H\left(Y^{*}, P_{t_{1}}^{s_{1}}\right)=0$ if $\operatorname{deg} P_{t_{1}}^{s_{1}}>\operatorname{deg} P_{t}^{s}\left(\operatorname{resp} . \operatorname{deg} P_{t_{1}}^{s_{1}}<\operatorname{deg} P_{t}^{s}\right)$.

The main result is the existence, uniqueness and naturality of these constructions.

TheOREM 3.3. (a) For any (b.b.) spectrum $X$ and any $P_{t}^{s}$ there are (b.b.) spectra of type $X\left\langle 0, P_{t}^{s}\right\rangle$ and of type $X\left\langle P_{t}^{s}, \infty\right\rangle$.

(b) If $Y_{1}$ and $Y_{2}$ are b.b. spectra of type $X\left\langle 0, P_{t}^{s}\right\rangle$ or $X\left\langle P_{t}^{s}, \infty\right\rangle$ then $Y_{1}$ and $Y_{2}$ are stably equivalent.

(c) If $f: X_{1} \rightarrow X_{2}$ is a map of spectra then there are spectra $Y_{1}$ and $Y_{2}$ of types $X_{1}\left\langle 0, P_{t}^{s}\right\rangle$ and $X_{2}\left\langle 0, P_{t}^{s}\right\rangle$, and a map $g: Y_{1} \rightarrow Y_{2}$ such that

$$
\begin{array}{ccc}
X_{1} & \stackrel{f}{\rightarrow} & X_{2} \\
\downarrow & & \downarrow \\
Y_{1} & \stackrel{g}{\rightarrow} & Y_{2}
\end{array}
$$

homotopy commutes. Similarly for type $X\left\langle P_{t}^{s}, \infty\right\rangle$. 
The same types of constructions can be performed for $A$-modules in the same way.

THEOREM 3.4. If $M$ is a realizable A-module then so are modules of types $M\left\langle 0, P_{t}^{s}\right\rangle$ and $M\left\langle P_{t}^{s}, \infty\right\rangle$.

Let me outline the proof of the existence of a spectrum of type $X\left\langle P_{t}^{s}+1, \infty\right\rangle\left(P_{t}^{s}+1\right.$ denotes the successor of $P_{t}^{s}$ in the ordering by degree). Let $A_{n}$ be the sub-Hopf algebra of $A$ generated by the first $n+1$ indecomposables: $P_{1}^{0}, P_{1}^{1}, \ldots, P_{1}^{n}$. Then $P_{t}^{s} \in A_{n}$ if and only if $s+t \leqq n+1$ and from this it follows that each $A_{n}$ contains an initial segment of the $P_{t}^{s}$ 's ordered by degree-specifically $\left\{P_{t}^{s} \mid \operatorname{deg} P_{t}^{s} \leqq \operatorname{deg} P_{n+1}^{0}\right\}$. Our first step is to prove the algebraic version of 3.3(a) for $A_{n}$-modules.

THEOREM 3.5. Given an $A_{n}$-module $M$ and $P_{t}^{s} \in A_{n}$ there is an $A_{n}$-module and monomorphism $f: M \rightarrow N$ such that

(a) $H\left(f, P_{t_{1}}^{s_{1}}\right)$ is an isomorphism if $\operatorname{deg} P_{t_{1}}^{s_{1}}>\operatorname{deg} P_{t}^{s}$,

(b) $H\left(N, P_{t_{1}}^{s_{1}}\right)=0$ if $\operatorname{deg} P_{t_{1}}^{s_{1}} \leqq \operatorname{deg} P_{t}^{s}$.

Further if $M$ is bounded below then so is $N$.

We construct $X\left\langle P_{t}^{s}+1, \infty\right\rangle$ as an inverse limit using

LEMMA 3.6. Let $X$ be a b.b. spectrum then there is a spectrum and map $f: Y \rightarrow X$ such that

(a) $H\left(f^{*}, P_{t_{1}}^{s_{1}}\right)$ is an isomorphism if $\operatorname{deg} P_{t_{1}}^{s_{1}}>\operatorname{deg} P_{t}^{s}$,

(b) if $\operatorname{deg} P_{t_{1}}^{s_{1}} \leqq \operatorname{deg} P_{t}^{s}$ and $H_{i}\left(X^{*}, P_{t_{1}}^{s_{1}}\right)=0$ for $i<w$ then $H_{i}\left(Y^{*}, P_{t_{1}}^{s_{1}}\right)$ $=0$ for $i \leqq w$.

In addition we can get $f$ to be a homotopy equivalence through $\operatorname{deg}(w+u)$ with $u$ independent of $w$.

OUTLINE OF PROOF. If $P_{t}^{s} \in A_{n}$ then regarding $X^{*}$ as an $A_{n}$-module we have by 3.5 the exact sequence of $A_{n}$-modules $0 \rightarrow X^{*} \stackrel{f}{\rightarrow} N \rightarrow L \rightarrow 0$ such that, for $P_{t_{1}}^{s_{1}} \in A_{n}$,

(a) $H\left(f, P_{t_{1}}^{s_{1}}\right)$ is an isomorphism if $\operatorname{deg} P_{t_{1}}^{s_{1}}>\operatorname{deg} P_{t}^{s}$ and

(b) $H\left(N, P_{t_{1}}^{s_{1}}\right)=0$ if $\operatorname{deg} P_{t_{1}}^{s_{1}}<\operatorname{deg} P_{t^{s}}^{s}$.

Let $F_{1} \stackrel{d}{\rightarrow} F_{2} \rightarrow L \rightarrow 0$ be an exact sequence of $A_{n}$-modules with $F_{1}$ and $F_{2}$ free. There are also maps $g: F_{1} \rightarrow X^{*}$ and $h: F_{2} \rightarrow N$ giving a commutative diagram. Then

$$
A \otimes_{A_{n}} F_{1} \stackrel{1 \otimes d}{\longrightarrow} A \otimes_{A_{n}} F_{2} \rightarrow A \otimes_{A_{n}} L \rightarrow 0
$$

is an exact sequence of $A$-modules and the map $g$ induces an $A$-map $g_{1}: A \otimes_{A_{n}} F_{1} \rightarrow X^{*}$

We now apply a well-known theorem and get $A \otimes_{A_{n}} F_{1}=K_{1}^{*}$, $A \otimes_{A_{n}} F_{2}=K_{2}^{*}$, and maps $K_{2} \rightarrow K_{1}$ and $X \rightarrow K_{1}$ inducing $1 \otimes d$ and $g_{1}$ in cohomology. Then $Y$ is defined as the pullback in 


$$
\begin{array}{ccc}
Y & \rightarrow K_{2} \\
\downarrow & \downarrow \\
X & \rightarrow K_{1} .
\end{array}
$$

If $Z \rightarrow K_{2} \rightarrow K_{1}$ is exact then so is $Z \rightarrow Y \rightarrow X$ and in addition it is possible to show that in this case $0 \rightarrow X^{*} \rightarrow Y^{*} \rightarrow Z^{*} \rightarrow 0$ is exact. So the lemma will be proved if we can show that $H\left(Z^{*}, P_{t_{1}}^{s_{1}}\right)=0$ for $\operatorname{deg} P_{t_{1}}^{s_{1}}>\operatorname{deg} P_{t}^{s}$ and that, for $i \leqq t, \delta: H_{i}\left(Z^{*}, P_{t_{1}}^{s_{1}}\right) \rightarrow H_{i+p}\left(X^{*}, P_{t_{1}}^{s_{1}}\right)(p$ $\left.=\operatorname{deg} P_{t_{1}}^{s_{1}}\right)$ is an isomorphism if $\operatorname{deg} P_{t_{1}}^{s_{1}} \leqq \operatorname{deg} P_{t}^{s}$. Let me indicate the argument used to prove the first of these assertions. Since $Z \rightarrow K_{2} h K_{1}$ is exact, $Z^{*}$ is an extension of coker $h^{*}=A \otimes{ }_{A_{n}} L$ by ker $h^{*}$. Recall that $0 \rightarrow X^{*} \rightarrow N \rightarrow L \rightarrow 0$ is exact, so using Theorem 2.3 we can show that $H\left(A \otimes_{A_{n}} L, P_{t_{1}}^{s_{1}}\right)=0$ if $\operatorname{deg} P_{t_{1}}^{s_{1}}>\operatorname{deg} P_{t}^{s}$. This implies the same for $\operatorname{ker} h^{*}$ since $0 \rightarrow \operatorname{ker} h^{*} \rightarrow K_{2} \rightarrow \operatorname{im} h^{*} \rightarrow 0$ and $0 \rightarrow \operatorname{im} h^{*} \rightarrow K_{1} \rightarrow \operatorname{coker} h^{*} \rightarrow 0$ are both exact. And therefore the exactness of $0 \rightarrow \operatorname{coker} h^{*} \rightarrow Z^{*} \rightarrow \operatorname{ker} h^{*}$ $\rightarrow 0$ gives us that $H\left(Z^{*}, P_{t_{1}}^{s_{1}}\right)=0$ if $\operatorname{deg} P_{t_{1}}^{s_{1}}>\operatorname{deg} P_{t}^{s}$. A more complicated argument is needed to prove the other assertion.

4. Postscript. As with the analogous work with homotopy groups, the results described in $\$ 3$ immediately imply a decomposition of arbitrary spectra into a tower of fibrations with fibre at each stage a spectrum whose cohomology has only one nonvanishing homology group. In a sequel to this paper I will describe my work with such spectra. Suffice it to say that they enjoy some very interesting properties.

\section{BIBLIOGRAPHY}

1: J. F. Adams and H. R. Margolis, Modules over the Steenrod algebra, Topology 4 (1971), 271-282.

2. E. H. Brown, Jr., On the realizability of $A / A\left\{\chi\left(S q^{i}\right) \mid i>k\right\}$ (unpublished).

3. E. H. Brown, Jr. and F. P. Peterson, $A$ spectrum whose $Z_{p}$-cohomology is the algebra of reduced pth powers, Topology 5 (1966), 149-154. MR 33 \# 719.

4. H. R. Margolis, Periodic spectra, Trans. Amer. Math. Soc. (to appear).

5. J. W. Milnor, The Steenrod algebra and its dual, Ann. of Math. (2) 67 (1958), 150-171. MR 20 \#6092.

Department of Mathematics, Boston College, Chestnut Hill, Massachusetts 02160 